\title{
PREVISÃO DOS IMPACTOS CAUSADOS POR POLUIÇÃO LUMINOSA COM ÊNFASE NOS SÍTIOS DE OBSERVAÇÃO ASTRONÔMICA E SÍNTESE DA PROPOSTA DE LEGISLAÇÃO NACIONAL
}

\section{PREDICTION OF THE IMPACTS CAUSED BY LIGHT POLLUTION WITH EMPHASIS IN OBSERVATIONAL ASTRONOMY SITES AND SYNTHESIS OF THE NATIONAL LEGISLATION PROPOSAL}

\section{Saulo Roberly Gargaglioni' ${ }^{1}$, Francisco Antônio Dupas², Alberto Rodriguez-Ardila ${ }^{3}$}

\footnotetext{
${ }^{1}$ Mestre em Engenharia da Energia pela Universidade Federal de Itajubá (UNIFEI). Membro no Núcleo de Estudos Ambientais, Planejamento Territorial e Geomática (NEPA) do Instituto de Recursos Naturais (IRN) da Universidade Federal de Itajubá (UNIFEI). e-mail: saulo@lna.br

${ }^{2}$ Docente/Pesquisador do Núcleo de Estudos Ambientais, Planejamento Territorial e Geomática (NEPA) do Instituto de Recursos Naturais (IRN) da Universidade Federal de Itajubá (UNIFEI). e-mail: dupas@unifei.edu

${ }^{3}$ Pesquisador do Laboratório Nacional de Astrofísica. e-mail: aardila@lna.br
}

\section{RESUMO}

São apresentados os resultados de (i) uma revisão bibliográfica sobre o tema, e a legislação existente no Brasil e de outros países que regulamentam o uso da iluminação artificial e, de maneira pioneira, (ii) uma proposta preliminar da legislação brasileira de disciplinamento e previsão dos impactos provocados em sítios de observação astronômica. Conclui-se que, além dos impactos ambientais, sociais e econômicos, a ciência astrofísica brasileira também sofrerá perdas significativas causadas pela poluição luminosa, mas, diante disso, é demonstrada uma síntese da proposta para criação de aparato legal. Destaca-se, também, a urgência da inclusão de tópicos específicos sobre a regulamentação nas normas ISO (International Organization for Standardization), na ABNT (Associação Brasileira de Normas Técnicas), no INMETRO (Instituto Nacional de Metrologia, Qualidade e Tecnologia) e na criação e na inserção de critérios de uso da iluminação artificial dentro do planejamento, da gestão territorial e urbana via planos diretores. Palavras-chave: Poluição luminosa. Impactos ambientais. Planejamento territorial e urbano. Uso racional da energia. Astronomia.

\section{ABSTRACT}

It present the results of (i) a literature review on the subject, and also the currently existing legislation in Brazil and other countries that regulate the use of artificial lighting, and in a pioneer 
way, (ii) a preliminary proposal of the Brazilian legislation for the disciplining and the predicting of the impacts caused on observational astronomy sites. It can be concluded that besides the environmental social and economical impact, it will also be extended to the Brazilian astrophysics science where you can see a synthesis of the proposal for the establishment of the legal apparatus. It can be also pointed out the urgency of including specific topics on the regulation of the ISO (International Organization for Standardization), the ABNT (Associação Brasileira de Normas Técnicas), the INMETRO (Instituto Nacional de Metrologia, Qualidade e Tecnologia) and also in the development and integration of the criteria for the use of artificial lighting in urban planning through directors plans.

Keywords: Light pollution. Environmental impacts. Urban planning. Energy rational use. Astronomy.

\section{INTRODUÇÃO}

Nas últimas décadas tem ocorrido um aumento crescente do brilho no céu noturno. Este acréscimo da luminosidade do céu noturno dos países é um dos efeitos mais notáveis da poluição luminosa. Esta pode ser definida como alteração nos níveis naturais de luz no ambiente externo, devido à iluminação artificial. $66 \%$ da população mundial e até $99 \%$ dos habitantes da América do Norte e Europa Ocidental não podem contemplar um céu estrelado devido à crescente contaminação visual provocada pela poluição luminosa no mundo.

Os benefícios da redução da poluição luminosa são no consumo energético, na proteção do meio ambiente noturno, na maior segurança no tráfego terrestre, marítimo e aéreo noturno, além da melhoria da qualidade das observações astronômicas. Comparando-se os mapas do brilho do céu com os dados da densidade populacional global do Departamento de Energia observa-se que cerca de $2 / 3$ da população mundial e $99 \%$ da população dos EUA (excluindo o Alaska e o Havaí) e a União Européia vivem em áreas onde o céu noturno está acima do limiar determinado para áreas poluídas. Adicionalmente, cerca de $1 / 5$ da população mundial, mais de $2 / 3$ da população dos EUA e mais da metade da população da União Européia perderam a visibilidade a olho nu da Via Láctea. No Chile, somente $1 / 7$ da população vive em áreas com céus não poluídos, enquanto mais da metade perdeu a possibilidade de ver a Via Láctea do lugar em que vivem. Cerca de 1/3 dos chilenos não podem adaptar totalmente seus olhos para visão noturna devido ao brilho do céu noturno (CINZANO et al., 2000; CINZANO, 2003).

Considera-se que o comprometimento da visibilidade do céu noturno não é causado somente pelas luminárias das vias públicas, mas também e principalmente, por outros fatores comuns aos grandes centros urbanos, como avisos luminosos, outdoors, quadras e estádios de futebol, iluminação de fachadas de prédios, monumentos, entre outros.

São três tipos principais de poluição luminosa: (i) o brilho no céu alaranjado que pode ser visto nas torres e cidades causado pelas luzes que se direcionam para a atmosfera, sendo refratadas e espalhadas pelas partículas ou aerossóis causados por poeira, pólen, bactérias, esporos, sal do mar, partículas minerais em suspensão dos desertos e produtos industriais; (ii) o ofuscamento que consiste na luz reluzindo para dentro dos olhos, impedindo a pessoa de enxergar, causando cegueira momentânea e trazer riscos para motoristas e pedestres e, (iii) a luz intrusa que brilha de um domínio para outro onde não é necessária, a exemplo das luzes de segurança que podem causar desconforto aos habitantes nas edificações ficando privadas da escuridão absoluta (HOUSE OF COMMONS, 2003).

Sistemas de iluminação mal projetados, direcionando a luz acima da linha do horizonte, podem ser evitados com planejamento e utilização adequada de lâmpadas, luminárias e acessórios (Figura1). Os dois primeiros exemplos são ineficientes, pois causam dispersão de luz acima da linha 
do horizonte. O brilho no céu tira a visão dos observadores e sistemas mais eficientes tornam o céu sem brilho.

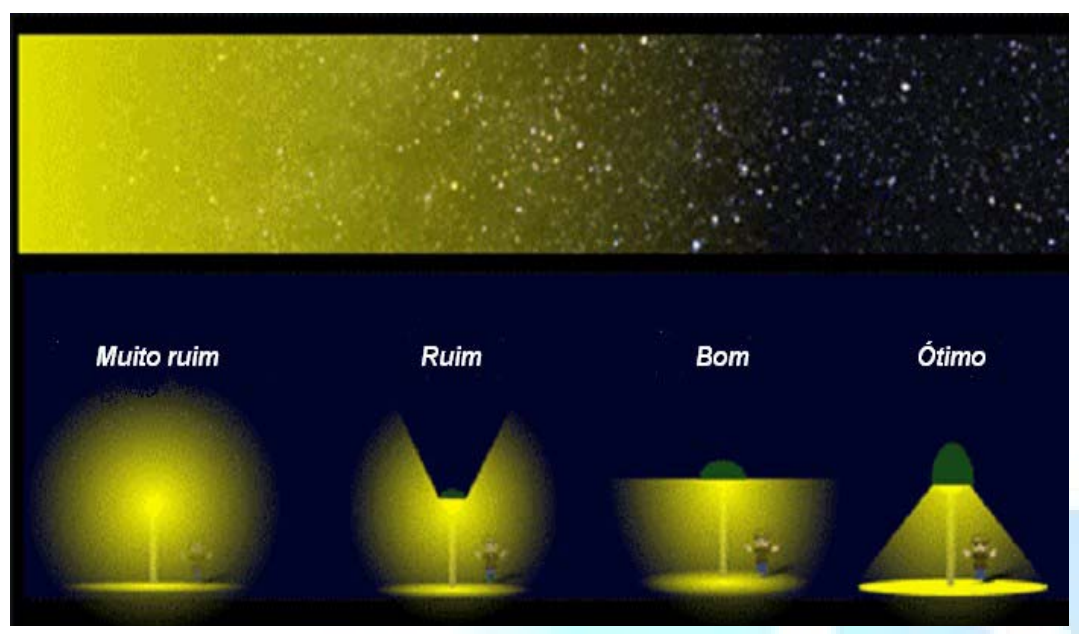

FIGURA 1. Planejamento de tipos de iluminação - escala de poluição luminosa em função do tipo de iluminação. Fonte: CSA (2012).

Os vários impactos ambientais causados pela poluição luminosa podem ocasionar mudanças na orientação e atração dos organismos em locais com iluminação ambiental alterada, podendo afetar a reprodução, migração e comunicação das espécies, além de estender comportamentos diurnos e crepusculares para o período noturno, por aumentar a habilidade do animal de se orientar, muitas vezes ampliando de aves e répteis (HILL, 1990; SCHWARTZ e HENDERSON, 1991; LONGCORE e RICH, 2004; SMITH, 2009).

Alguns invertebrados e vertebrados que são atraídos pela iluminação do parceiro perdem a capacidade de reprodução e deslocamento. Canhões de luz lançados diretamente ao céu ocasionam problemas na migração das aves, sendo causa de grande mortalidade pela perda de orientação e batendo em obstáculos. Pássaros atraídos pela luz dos prédios, torres de transmissão, monumentos e outras construções, voam sem cessar em torno da luz até caírem de cansaço (SALMON et al., 1995; CHARRO, 2001; LONGCORE e RICH, 2004).

$\mathrm{Na}$ flora, um dos principais efeitos é que as plantas não florescem se a duração da noite é mais curta do que o período normal, enquanto outras florescerão prematuramente como resultado da exposição ao fotoperíodo necessário para o florescimento, na diminuição de insetos que realizam a polinização, crescimento anormal e defasagem nos períodos de floração (CHARRO, 2001; HOUSE OF COMMONS, 2003).

Pela ampliação da demanda por geração de energia, também crescem as emissões de dióxido de carbono, aumento de temperatura pelo uso de combustíveis fósseis e produção de lâmpadas e seus impactos derivados da sua produção como substâncias de mercúrio, antimônio, bário, chumbo, cádmio, índio, sódio, estrôncio, tálio, vanádio e ítrio (NARISADA e SCHREUDER, 2004; NETRESIDUOS, 2007).

Os impactos da poluição luminosa sobre a saúde humana são relevantes. Motoristas podem ter sua capacidade visual reduzida e, a invasão da luz nas casas pode prejudicar a qualidade do sono das pessoas e ocasionar estresse, desvalorizar imóveis, podendo ser um fator de risco para o câncer de mama, devido à supressão da luz noturna sobre a glândula pineal, reduzindo a produção de hormônios sintetizados no escuro (REITER, 1991; BRAINARD et al, 1997; DAUCHY et al., 1997; ARENDT, 1998; CRAWFORD e GENT, 2002; BLASK et al., 2002; GLICKMAN et al., 2002; CLAUDIO, 2009; CHEPESIUK, 2009). 
As primeiras iniciativas legislativas em poluição luminosa que surgiram nos Estados Unidos foram resultado da pressão exercida pela International Dark Sky Association (IDA). De 1992 até 1993, a cidade de Tucson, no Arizona, trocou as lâmpadas de mercúrio por vapor de sódio. Cerca de 40.000 lâmpadas foram instaladas direcionadas para o solo, reduzindo o brilho no céu noturno e reduzindo os custos em cerca de dois milhões de dólares. Além das doenças consequentes da poluição luminosa e do alto custo proporcionado, por exemplo, nos EUA cerca de 2 bilhões de dólares são desperdiçados anualmente com a iluminação ineficiente (CHARRO, 2001; CRAWFORD e GENT, 2002; DEL CASTILLO et al., 2003; IDA, 2006).

Na Itália, nas Províncias de Frosinone e Ferentino, após adoção de regulamentação de modificação na iluminação no ano 2000, os resultados com redução de consumo de energia foram de aproximadamente de 28 milhões de lumens o que equivale passar de $27,45 \mathrm{~kW} / \mathrm{h}$ para $15,75 \mathrm{~kW} / \mathrm{h}$ sendo ampliada em 50\% após as 23:00 horas. Isso provocou economia de 9.202 euros. O custo para implantação do sistema foi de 8.780 euros em 2001 e foi pago em um ano (DI SORA, 2002).

$\mathrm{Na}$ Hungria, para prevenir o crescimento da degradação da visualização do céu noturno foi criada uma associação no ano 2000. As metas com a reconstrução do sistema de iluminação das estações ferroviárias foram atingidas. $O$ resultado foi uma redução de $40 \%$ no consumo de energia elétrica. A economia foi maior que 1 milhão de euros por ano e o custo total da reconstrução foi recuperado em 3 anos (KOLLÁTH, 2002).

Nas Ilhas Canárias a lei de proteção da qualidade astronômica dos observatórios foi votada em 1992. Os resultados mostram que o consumo anual de energia foi de $368 \mathrm{MWh} /$ ano com um custo de 33.169 euros/ano, baixou para $80 \mathrm{MWh} /$ ano custando 7.212 euros/ano. Chegaram a uma economia no consumo de energia de 65\% (CASTRO e LA PAZ, 2003; CASTRO, 2006).

O Chile apresenta uma legislação nacional para o controle da qualidade da iluminação, de diminuição do gasto energético e brilho no céu noturno desde o final da década de 90 , sendo os observatórios beneficiados por essas medidas. Em Monte Pátria foi instalado um sistema que reduz o consumo em $60 \%$, e a emissão de luz em $36 \%$, após 4 horas de uso. A economia de energia foi de US\$ 150,000/ano, sendo observadas melhorias na segurança pública (SANHUEZA e SANTANDER, 2003).

A legislação brasileira com relação à poluição luminosa é pequena. Foram constatadas apenas quatro legislações sendo do IBAMA, a Lei Estadual da Bahia, da prefeitura de Campinas (SP), de Caeté (MG) e algumas citações na ABNT e na ISO 14001. A Portaria IBAMA n 11 de 1995 protege toda a costa brasileira onde há desova de tartarugas (IBAMA, 2006) via Projeto TAMAR que estabelece alguns pontos frágeis a serem cuidados e a Lei Estadual da Bahia $n^{\circ} 7034$, de 1997 (GODOY, 2003 apud GARGAGLIONI, 2007).

O Observatório Municipal de Campinas Jean Nicolini foi inaugurado em 15 de janeiro de 1977. Suas ações abrangem atividades educativas, de divulgação e pesquisa astronômica. A Lei $\mathrm{n}^{\circ}$ 10.850/2001 cria a Área de Proteção Ambiental - APA - do município de Campinas, onde está localizado o Observatório (CAMPINAS, 2007).

O Observatório Astronômico da Serra da Piedade, localizado na Serra da Piedade, na cidade de Caeté e ligado a Universidade Federal de Minas Gerais - UFMG foi inaugurado em 1973. A legislação foi aprovada pela Câmara de Caeté próximo ao ano de 1982 (TARSIA, 2007 apud GARGAGLIONI, 2007).

A ABNT normatiza as técnicas no país. Pode padronizar materiais que podem resultar na diminuição da poluição luminosa e fixa requisitos mínimos necessários à iluminação de vias públicas, à segurança ao tráfego de pedestres e veículos, à iluminação de túneis, de luminárias para iluminação pública, de lâmpadas a vapor de sódio a alta pressão e a vapor metálico e reator e ignitor para lâmpada a vapor de sódio a alta pressão (ABNT, 1976; 1992; 2003; IEC, 1997a; 1997b; 1999; 2004; BRASIL, s/d). 
A ISO 14001 busca abranger todas as situações que possam interagir de forma negativa com o meio ambiente e propor atividades de gerenciamento cabíveis. No caso da poluição luminosa, que afeta o comportamento de animais e seus hábitos, a ISO 14001 poderia colaborar na minimização destes impactos, contudo, são pouco citados nos Sistemas de Gestão Ambiental brasileiro (NEVES, 2007 apud GARGAGLIONI, 2007).

Também para a astronomia, a luz que é direcionada para o espaço é prejudicial, pois uma parte desta luz é refletida por gotículas formadas pela umidade e partículas de pó atmosféricas, causando um fundo luminoso que sobrepõe à luz natural do céu e das estrelas (MIRANDA, 2003; BENN, 2008). A redução da luz natural trás sérias perdas financeiras devido aos investimentos realizados em centros de pesquisa de estudos em astronomia. Outro estudo que se baseou em um telescópio de 8 metros com custo de aproximadamente 85 milhões de dólares constatou que um aumento de $25 \%$ na iluminação noturna ocasiona uma perda de quase 20 milhões de dólares para a astronomia, sendo que este valor representa aproximadamente 2,5 vezes o aporte total do Chile para o Gemini Observatory (MENDEZ e SCHMIDT, 2012).

Assim, identifica-se que para o Brasil, onde esse problema tem sido muito pouco considerado, existem poucos locais com regulamentações sobre o assunto. De maneira que, a presente pesquisa vai de encontro aos interesses e a necessidade de criar um aparato legal brasileiro. A iluminação excessiva deve ser considerada poluente por ser causadora de danos ambientais, sociais, econômicos e científicos e deve ser tratada como um problema que necessita ser regulamentado com urgência. Também, por meio de uma simulação foi realizada uma previsão de perda da capacidade de observação do céu utilizando o sítio astronômico do Pico dos Dias localizado em Brazópolis (MG), que pertence ao Laboratório Nacional de Astrofísica - LNA.

\section{MATERIAL E METODOS}

Como estudo de caso, foi utilizado o sítio de observação astronômica do Laboratório Nacional de Astrofísica (LNA), denominado por Observatório Pico dos Dias (OPD). Esta é uma das unidades de pesquisa integrantes da estrutura do Ministério da Ciência e Tecnologia - MCT desde 1985 onde é disponibilizado um telescópio de 1,6m Perkin-Elmer. Está localizado no sul do Estado de Minas Gerais com sua sede na cidade de Itajubá, com o sítio do telescópio na cidade de Brazópolis (Figura 2).

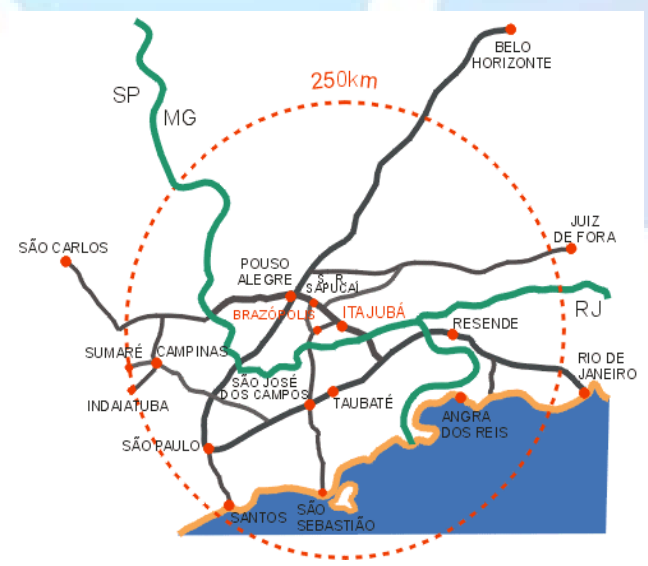

FIGURA 2. Mapa de localização do OPD e das cidades de Itajubá e Brazópolis (LNA, 2007). 
Os resultados do estudo de simulação dos impactos da poluição luminosa sobre o OPD, dentre eles os ambientais, sociais, econômicos norteados pela legislação brasileira, estão fundamentados no estado da arte apresentado na introdução deste artigo. São apresentados estudos de sistemas de prevenção no Brasil e no mundo por meio de experiências internacionais. Os resultados desta proposta de avaliação dos impactos poderão ser usados nos sítios de observação astronômica, especificamente o OPD, pois estão diretamente relacionados com a qualidade das observações que, por sua vez, está diretamente relacionada à quantidade de brilho do céu. De maneira que o impacto sobre a astronomia pode ser demonstrado por meio da Equação (1) (WALKER, 1970).

$$
\mathrm{I}=0,01 \cdot \mathrm{P} \cdot \mathrm{d}^{-2,5}
$$

Onde: I é o aumento do nível de brilho do céu acima do céu escuro natural; P é a população da cidade em habitantes; $d$ é a distância do centro da cidade em km; 0,01 é uma constante típica para a maioria das cidades que apresentam uma determinada quantidade de iluminação pública.

Inicialmente, a lei foi desenvolvida para cidades da Califórnia (EUA). Em uma primeira aproximação acredita-se que pode ser aplicada a outros observatórios. A Lei de Walker pode ser usada para estimar o brilho do céu em um sítio de observação com o telescópio em um ângulo a $45^{\circ}$ de zênite em direção a uma fonte urbana a "d" quilômetros de distância (IDA, 2006; 2007a, 2007b). Como exemplo, I=0,02 significa ganho de $2 \%$ no brilho do céu quando comparado ao céu escuro sem a contribuição de fontes artificiais. Se $\mathrm{I}=1,0$ significa que o brilho do céu é o dobro do fundo do céu escuro natural, um aumento de $100 \%$. Esta equação enquadra-se melhor em cidades onde a quantidade de lumens emitidos por pessoa fica entre 500 e 1000 (aproximadamente equivalente a uma lâmpada de 30 e 60 watts, respectivamente). Já em grandes cidades, onde a quantidade de lumens emitidos por a pessoa é maior, o aumento do brilho no céu indicado pela fórmula pode ser maior do que o real.

A aproximação e a correlação das perdas econômicas para a astronomia e para o OPD foi baseada em Crawford (2000), que desenvolveu um método fundamentado em variáveis como a dimensão do espelho do telescópio e o nível de brilho do céu. Na Tabela 1 é mostrado como uma pequena quantidade de poluição luminosa pode afetar o poder de observação de um telescópio, acarretando assim em perda econômica para a astronomia e para os observatórios. A abertura equivalente $\left(\mathrm{D}_{\mathrm{E}}\right)$ do telescópio foi definida na Equação (2):

$$
D_{E}=D / X^{1 / 2}
$$

Onde: D é o diâmetro do espelho primário e X significa o nível de brilho do céu aumentado quando comparado ao fundo de céu sem a contribuição de iluminação artificial.

TABELA 1 - Valor perdido em um telescópio de 4 metros no diâmetro do espelho devido ao aumento do brilho no céu (adaptado de CRAWFORD, 2000).

\begin{tabular}{cc}
\hline Brilho do céu (X) & $\begin{array}{c}\text { Diâmetro do espelho em } \\
\text { metros (D) }\end{array}$ \\
\hline 1,00 & 4,00 \\
\hline 1,10 & 3,81 \\
\hline 1,20 & 3,65 \\
\hline 1,25 & 3,58 \\
\hline 1,50 & 3,27 \\
\hline 2,00 & 2,83 \\
\hline 3,00 & 2,31 \\
\hline 4,00 & 2,00 \\
\hline
\end{tabular}


Para um céu sem contribuição de luz feita pelo homem, tem-se um valor de $X=1,00$. Um valor de $X=1,20$ significa um aumento de $20 \%$ no brilho do céu, devido à produção de poluição luminosa pelo homem. No caso de um telescópio de 4 metros e $X=1,20$ significa diminuir o diâmetro efetivo deste telescópio para um de 3,81 metros. Para um valor de $X=2,00$, significa duplicar o nível da luminosidade natural do céu, transformando um telescópio de 4 metros em um com desempenho equivalente àquele de 2,83 metros e assim por diante.

No estudo do caso do OPD foi elaborada uma previsão dos impactos causados pelas cidades existentes no entorno do sítio. Foram apresentados e analisados os resultados sobre a perda da capacidade de observação do OPD. Ressalta-se que as predições necessitam ser confirmadas experimentalmente e, os valores anotados, por utilizar modelos de fora do Brasil, podem ser considerados resultados aproximados. Um tratamento mais rigoroso do assunto requer medições in loco para determinar as perdas reais por conta da poluição luminosa. Adicionalmente, são apresentadas técnicas de prevenção, onde se comprova a mudança nos sistemas de iluminação e os ganhos por ela realizados. Nas conclusões são mostradas as necessidades de implementação da legislação nacional sobre fixação de padrões de iluminação e as vantagens com a economia de energia e eficácia que a mudança de sistemas de iluminação hoje existentes poderá trazer.

\section{RESULTADOS E ANÁLISES}

Tomando a Lei de Walker (Equação 1) e as cidades existentes no entorno do OPD se como exemplo e seus correspondentes parâmetros listados na TABELA 1, obtém-se o gráfico da Figura 3. Na Tabela 2 são mostradas as distâncias e a população de cada cidade do entorno do OPD. Nos cálculos, foi considerado que a faixa de lumens emitidos por pessoa está entre 500-1000. A distância (d) é dada em km, em linha reta entre cada cidade do entorno do OPD. Os resultados mostram que para a cidade de Itajubá, $I=0,66$. Isto significa que a cidade de Itajubá contribui com $66 \%$ de aumento no brilho do céu comparado com o fundo natural. Vale destacar que esta simulação utiliza padrões de iluminação norte-americanos, local onde foram considerados os valores da faixa de lumens emitidos, portanto, podem ser diferentes dos instalados no Brasil.

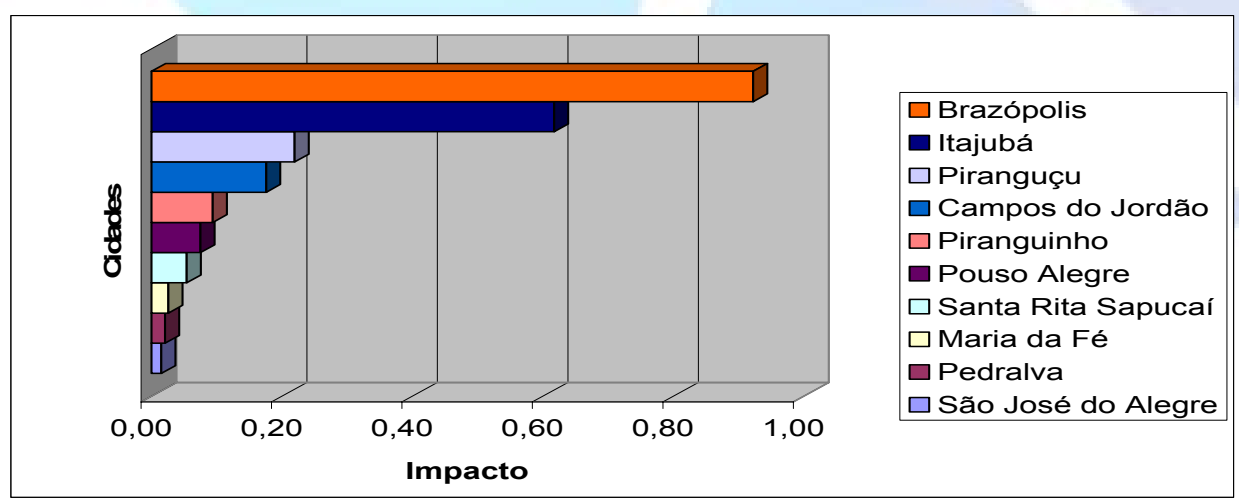

FIGURA 3. Resultados do impacto da poluição luminosa determinado por cada cidade. 
TABELA 2 - Distâncias e a população de cada cidade do entorno do OPD.

\begin{tabular}{crr}
\hline Cidade & D & \multicolumn{1}{c}{ P } \\
\hline São José do Alegre & 24.0 & 4.178 \\
Pedralva & 33.0 & 12.756 \\
Maria da Fé & 32.5 & 15.330 \\
Santa Rita do Sapucaí & 33.5 & 34.920 \\
Pouso Alegre & 49.0 & 125.209 \\
Piranguinho & 15.0 & 8.160 \\
Campos do Jordão & 24.0 & 49.512 \\
Piranguçu & 9.0 & 5.329 \\
Itajubá & 18.5 & 90.812 \\
Brazópolis & 7.5 & 14.206 \\
\hline
\end{tabular}

$\mathrm{D}=$ distância em km e $\mathrm{P}=$ população.

Apesar da cidade de Itajubá ser maior do que a cidade de Brazópolis, o impacto provocado por Brazópolis é maior $(\mathrm{I}=0,92)$ visto que se encontra a $7,5 \mathrm{~km}$ do OPD e Itajubá a $18,5 \mathrm{~km}$, mesmo que esta seja 6,4 vezes maior que a referida cidade.

Crawford (2000) demonstrou por meio da Equação (2), que o aumento da luminosidade artificial do céu reduz o poder de observação dos telescópios e exemplificou a questão em um telescópio de $4 \mathrm{~m}$. O poder de observação é reduzido à medida que a porcentagem do fundo do céu foge do valor natural, como se o diâmetro do espelho fosse reduzido. Na Figura 4 são mostrados os resultados obtidos da aplicação da Equação (1) ao telescópio de 1,60 m instalado no OPD. Identifica-se que as alterações preditas no fundo de céu natural provocam perdas no poder de observação dos telescópios resultando em uma "redução aparente" do diâmetro efetivo do espelho para 1,13 m quando a contribuição de luz artificial duplicar em relação a um céu sem luz artificial, ou seja, sem poluição luminosa.

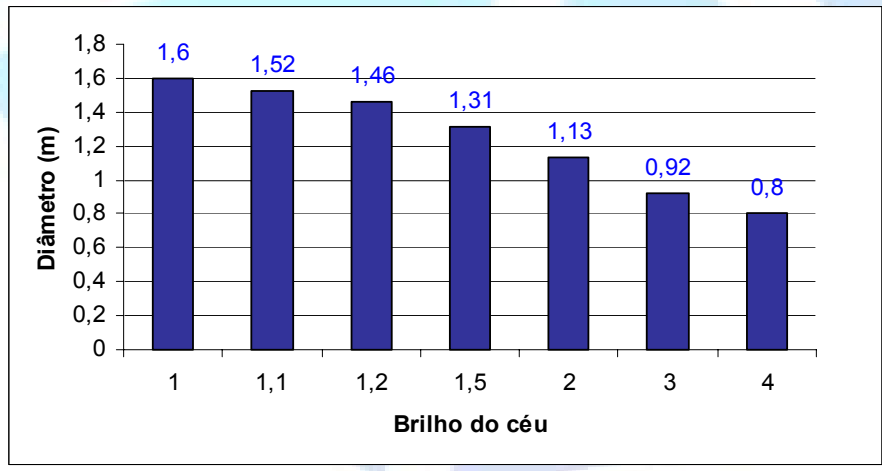

FIGURA 4. Diâmetro do espelho x Brilho do céu.

Este brilho excessivo é indicativo da falta de planejamento dos sistemas de iluminação causado por toda luz que ilumina acima da linha do horizonte. Deve-se considerar que muitos pontos de iluminação são obsoletos, alguns ainda utilizam lâmpadas incandescentes e muitos outros utilizam as lâmpadas de vapor de mercúrio, que são altamente poluentes em todo o espectro visível.

É importante salientar também o fator angulação nos sistemas de iluminação. Além de o sistema ter que ser moderno e de alto rendimento luminotécnico, o ângulo de instalação deve ser considerado e instalado a 90 graus em relação ao plano em que está fixado para que não ilumine acima da linha do horizonte. A propagação cada vez maior da poluição luminosa constitui em um 
injustificável gasto de energia, provoca desequilíbrio do ecossistema, interfere na qualidade e na saúde humana e impede a observação do céu pela população e pelos cientistas.

Os impactos sociais relacionados ao excesso de iluminação também poderiam ser reduzidos com regulamentações nos níveis de iluminação evitando-se tanto o ofuscamento da visão dos seres humanos quanto à fadiga visual, melhorando a percepção visual e a segurança pública e nas rodovias. Destaca-se ainda que estas regulamentações devam resultar em projeto de lei, pois evitariam a intensidade de luz muito forte que invade as casas e que podem causar problemas no sono e estresse. Uma correlação muito importante a ser considerada é a de que a exposição à luz durante a noite pode aumentar o risco do câncer de mama.

Da mesma forma que existem áreas de proteção sonora, é necessário existir áreas de proteção de iluminação, onde se incluem os observatórios astronômicos, áreas de proteção ambiental (APA), reservas naturais, entre outras; onde ocorreriam espaços estritamente reservados para pesquisas astronômicas científicas e áreas recreativas para observação das estrelas. A pesquisa científica na área da astronomia também é prejudicada pelo excesso de iluminação artificial durante a noite. Como ocorre em outros países, incluindo o Brasil, a falta de planejamento nos sistemas de iluminação nas cidades limita o poder de observação dos telescópios pela perda do poder de observação dos mesmos, prejudicando as pesquisas cientificas.

A indução a uma proposta preliminar de legislação brasileira que visa disciplinar o uso da iluminação artificial tendo como base a ordenação ambiental dos sistemas de iluminação para a proteção de meio noturno, foi fundamentada a partir da legislação da Catalunia, Espanha (MURIANA, 2008a, 2008b). Sucintamente, esta tem como objeto a regulamentação de instalações de iluminação externas e internas, públicas e privadas, com intuito de se evitar a poluição luminosa que ocorre devido ao mau planejamento dos sistemas de iluminação e dos efeitos produzidos por ela. Em sua essência, a legislação brasileira deverá ser composta por: a) Regular a implantação dos sistemas de iluminação; b) Manter as condições naturais ao máximo possível; c) Promover a eficiência energética e luminosa das iluminações externas e internas; d) Evitar a luz intrusa nas residências; e) Prevenir e corrigir os efeitos da poluição luminosa na visão do céu. Maiores detalhes da proposta poderão ser encontrados em Gargaglioni (2007).

\section{CONCLUSÕES}

O excesso de iluminação não pode ser apontado como qualidade de vida para os cidadãos. Projetos mal elaborados e que são responsáveis por um gasto energético desnecessário influenciam negativamente em aspectos sociais importantes, tais como a preservação da saúde e intimidade familiar, assim como afetam a visibilidade dos objetos celestes na superfície terrestre. É possível de ver o céu e as estrelas em seu modo natural, sem a existência de poluição luminosa no contexto de um meio ambiente adequado, considerando ainda a observação dos corpos celestes na área de pesquisa cientifica.

Neste estudo, é possível concluir que as iniciativas brasileiras, tanto no plano nacional, estadual e municipal são ainda pequenas e há muito a ser realizado. Portanto, torna-se importante e urgente elaborar uma proposta de legislação brasileira pertinente que discipline os sistemas de iluminação públicos e privados, de modo a reduzir a poluição luminosa. A adoção de planejamentos mais criteriosos e modernos possibilita cidades mais eficientemente iluminadas e a mitigação dos danos ambientais sociais e ambientais.

Os dados levantados dos diversos países pesquisados demonstram uma relação entre um menor consumo de energia, maior quantidade de lumens emitidos, redução na potência instalada e menor fluxo de luz direcionado ao céu quando as medidas de eficiência nos sistemas de iluminação são implementadas. 
Sugere-se que as normas da série ISO 14000, a ABNT e INMETRO podem ter importante papel também nesta normatização e na minimização de impactos ambientais e na pesquisa astronômica causados pela poluição luminosa, visto que pode ser encarada como um aspecto ambiental a ser gerenciado através da legislação específica, apesar de não citado nos Sistemas de Gestão Ambiental (SGA) brasileiros. A proposta completa de legislação nacional sobre o tema pode ser encontrada em Gargaglioni (2007).

Além das normas, poderá ser implementada legislação específica nos municípios via planos diretores urbanos, como é o caso pioneiro de Santa Rita do Sapucaí, MG. Neste município foram estabelecidas diretrizes de maneira a disponibilizar aos munícipes o zoneamento de áreas susceptíveis que busquem minimizar os efeitos causados pela poluição luminosa tanto internos quanto no entorno por ser uma área no entorno do Observatório Nacional de Astrofísica (DUPAS, 2009).

Por fim, considera-se importante que novos estudos sejam realizados visando aprofundar e detalhar o conhecimento científico brasileiro na área em questão.

\section{REFERÊNCIAS}

ABNT - ASSOCIAÇÃO BRASILEIRA DE NORMAS TÉCNICAS. NBR 15129. Luminárias para iluminação pública. Requisitos Particulares. 2004. Disponível em:

http://www.abntcatalogo.com.br/norma.aspx?ID=320. Acesso em: 20 nov. 2006.

. NBR 13593. Reator e ignitor para lâmpada a vapor de sódio a alta pressão - Especificação e ensaios. 2003. Disponível em: http://www.abntcatalogo.com.br/norma.aspx?ID=4460. Acesso em: 20 nov. 2006.

. NBR IEC 60598. Luminárias. 1999. Disponível em:

http://www.abntcatalogo.com.br/norma.aspx?ID=002804. Acesso em: 20 nov. 2006.

. NBR 5123. Relé fotelétrico e tomada para iluminação - Especificação e método de ensaio. 1998. Disponível em: http://www.abntcatalogo.com.br/norma.aspx?ID=4685. Acesso em: 20 nov. 2006.

. NBR IEC 60662a. Lâmpadas a vapor de sódio a alta pressão. 1997. Disponível em: http://www.abntcatalogo.com.br/norma.aspx?ID=4277 . Acesso em: 20 nov. 2006.

. NBR IEC 1167b. Lâmpadas a vapor metálico (halogenetos). 1997. Disponível em: http://www.4shared.com/office/Z mDpjwm/nbr iso iec 1167 - lampadas a .html. Acesso em: 20 nov. 2006.

. NBR 5101: fixa requisitos, considerados como mínimos necessários, à iluminação de vias públicas, os quais são destinados a propiciar algum nível de segurança aos tráfegos de pedestres e veículos. 1992. Disponível em: http://www.abntcatalogo.com.br/norma.aspx?ID=4551 . Acesso em: 20 nov. 2006.

NBR 5181. Iluminação de túneis. Requisitos mínimos necessários à obtenção de uma visibilidade imediata, acurada e confortável para obras de arte especiais, durante os períodos diurno e noturno. 1976. Disponível em: http://www.abntcatalogo.com.br/norma.aspx?ID=9390. Acesso em: 20 nov. 2006. 
ARENDT, J. Melatonin and the pineal gland: influence on mammalian seasonal and circadian physiology. Journals of Reproduction and Fertility, v.3, p. 13-22, 1998.

BENN, C. R. Measuring Light Pollution on La Palma. Edited by Cipriano Marín and Jafar Jafari StarLight A Common Heritage. Published by Starlight Initiative Instituto de Astrofísica de Canárias (IAC), p. 435-441, 2008.

BLASK, D. E; SAUER, L.A; DAUCHY, R.T. Melatonin as a chronobiotic/anticancer agent: cellular, biochemical, and molecular mechanisms of action and their implications for circadianbased cancer therapy. Current Topics in Medicinal Chemistry, v. 2, n. 2, p. 113-32, 2002.

BRAINARD, G. C.; ROLLAG, M. D.; HANIFIN, J.P. Photic regulation of melatonin in humans: ocular and neural signal transduction. Journal of Biological Rhythms, v. 12, n. 6, p. 537-546, 1997.

CAMPINAS. Lei Municipal no 10.850 de 07 de junho de 2001. Legislações. Disponível em: http://www.campinas.sp.gov.br . Acesso em: 18 mai. 2007.

CASTRO, J. D. The sky law: problems and benefits in its application. Oficina técnica para la protección de calidad del cielo. Instituto de Astrofísica de Canárias, 2006.

CASTRO, J. D.; DE LA PAZ, F. The Law of the heavens of the Canaries. In: Light Pollution: The Global View. SCHWARZ, H.E.- Editor. La Serena, Chile. 2003.

CHARRO, M. C. El derecho a ver las estrellas: Análisis de la contaminación lumínica desde una perspectiva jurídica. Revista de derecho urbanístico y medio ambiente, Año $n^{\circ} 35, n^{\circ} 187$, p. 131-181, 2001.

CHEPESIUK, R. Missing the Dark. Health Effects of Light Pollution. Environmental Health Perspectives, v. 117, n.1, A20-A27, 2009.

CINZANO, P. Light pollution and the situation of the night sky in Europe, In Italy and in Veneto. Proceedings of the IDA Regional Meeting "Venice: Let's save to night". In: CINZANO, P. (Ed.), Light Pollution Science and Tecnology Institute. Thiene, Itália, v.4, 2003.

CINZANO, P.; FALCHI, F.; ELVIDGE, C.D.; BAUGH, K.E. The artificial night sky brightness mapped from DMSP satellite Operational Linescan System Measurements. Monthly Notices of the Royal Astronomical Society, v.318, p. 641-657, 2000.

CLAUDIO, L. Policies for Smarter Lighting. Environmental Health Perspectives, v. 117, n. 1, A28-A31 p., 2009.

BRASIL (s/d). Legislação - Resoluções CONAMA. Disponível em:

$<$ http://www.mma.gov.br/conama>. Acesso em: 14 mar 2010.

CRAWFORD, D. L.; GENT, R. L. Progress and Challenges in Light Pollution Control. Proceedings of the IDA Regional Meeting "Venice: Let's save to night". In: CINZANO, P. (Ed.) Light pollution and the protection of the night environment. Light Pollution Science and Technology Institute. Padova, Itália, p. 69-74, 2002. 
CRAWFORD, D.L. Light Pollution, an environment problem for astronomy and for manking. Measuring and modeling light pollution. Memorie della Società Astronomia Italiana, vol. 71-1, p.11-40. NASA Astrophysics Data System, Padova, Itália. 2000.

CSA - CANADIAN SPACE AGENCY. What is Light Pollution? (2012). Disponível em: http://www.asc-csa.gc.ca/eng/educators/resources/stars/light.asp. Acesso em: 18 mar. 2012.

DAUCHY, R.T.; SAUER, L.A.; BLASK, D.E.; VAUGHAN, G.M. Light contamination during the dark phase in "photoperiodically controlled" animal rooms: effect on tumor growth and metabolism in rats. Laboratory Animal Science - PubMed. National Center for Biotechnology Information, U.S. National Library of Medicine, Outubro, v. 47, n.5, 511-518 p., 1997.

DEL CASTILLO, E. M. A.; CRAWFORD, D. L.; DAVIS, D. R. Preserving our nighttime environment: a global approach. In: SCHWARZ, H.E. Light Pollution: The Global View. (Ed.) Cerro Tololo Inter-American Observatory, NOAO, La Serena, Chile. Astrophysics and Space Science Library, Kluwer Academic Publishers, Dordrecht, v. 284, 49 p., 2003.

DI SORA, M. Light pollution and energy saving: excellent results obtained in the application of new regulations. Proceedings of the IDA Regional Meeting "Venice: Let's save to night". In: CINZANO, P. (Ed.) Light pollution and the protection of the night environment. Light Pollution Science and Technology Institute. Padova, Itália. , p. 177-181, 2002.

DUPAS, F. A. Plano Diretor Participativo de Santa Rita do Sapucaí, MG. Relatórios finais de Projeto de Pesquisa CNPq Edital MCT/Mcidades n. 060/2005 proc. n. 501840/2005-20, v. 2, Disponível em: http://www.pmsrs.mg.gov.br., v.1, 160 p., v.2, 83 p., 2009.

GARGAGLIONI, S. R. Análise Legal dos Impactos Provocados pela Poluição Luminosa do Ambiente Urbano. 118f. 2007. Dissertação (Mestrado em Engenharia da Energia) - Instituto de Recursos Naturais, Núcleo de Estudos Ambientais, Planejamento Territorial e Geomática - NEPA, Universidade Federal de Itajubá, 2007. Disponível em: http://adm-net-a.unifei.edu.br/phl/pdf/0032988.pdf. Acesso em: 27 abr. 2007.

GLICKMAN, G; LEVIN, R.; BRAINARD, G. C. Ocular Input for Human Melatonin Relevance to Breast Cancer. Neuroendocrinology Letters. Filadelfia, Estados Unidos, jul, v.23, Suppl. 2, p.1722, 2002.

GODOY, M.L. A técnica de mitilicultura na preservação de tartarugas marinhas na comunidade de pescadores da Praia de Almada-Ubatuba-SP: um estudo de caso. 120 f. 2003. Dissertação (Mestrado em Tecnologia) Centro de Federal de Educação Tecnológica do ParanáCEFET-PR. Curitiba, Paraná, 2003.

HILL, D. The impact of noise and artificial light on waterfowl behavior: a review and synthesis of the available literature. British Trust for Ornithology Research Report No. 61 Norfolk, United Kingdom, v.5, 20 p., 1990.

HOUSE OF COMMONS. Light Pollution and Astronomy. Science and Technology Committee Publications, Seventh Report of Session 2002-03, Londres, Inglaterra, v. 1, 73 p., 2003. 
IBAMA. Legislação - Portaria IBAMA n 11 de 30 de janeiro de 1995. Disponível em: http://ibama2.ibama.gov.br/cnia2/renima/cnia/lema/lema_texto/IBAMA/PT0011-300195.PDF, Acesso em: 20 nov. 2006.

IDA - INTERNATIONAL DARK-SKY ASSOCIATION - Disponível em: http://www.darksky.org/assets/documents/Lighting\%20Code\%20Handbook.pdf. 73 p. Acesso em: 20 nov. 2006.

IDA - INTERNATIONAL DARK-SKY ASSOCIATION. Disponível em: http://www.darksky.org/assets/documents/Pima\%20County\%20Report.pdf. 20 p. Acesso em: 10 jun. 2007.

ISO - INTERNATIONAL ORGANIZATION FOR STANDARDIZATION. ISO 14001, Disponível em: http://www.abnt.org.br/m3.asp?cod_pagina=1130. Acesso em: 20 jun 2007.

KOLLÁTH, Z. Towards limiting light pollution in Hungary. Proceedings of the IDA Regional Meeting "Venice: Let's save to night". In: CINZANO, P. (Ed.) Light pollution and the protection of the night environment. Light Pollution Science and Tecnology Institute. Pad ova, Itália. , p. 291-299, 2002.

LNA - LABORATÓRIO NACIONAL DE ASTROFÍSICA. Mapa de localização do Observatório. Disponível em: http://www.lna.br. Acesso em: 6 jun 2007.

LONGCORE, T.; RICH, C. Ecological light pollution. The Ecological Society of America, Front Environment and Ecology; vol.2, n.4, p.191-198, 2004.

MENDEZ, R.; SCHMIDT, R. La Contaminación Lumínica: ¿Cómo Afecta a la Astronomía? National Optical Observatores. La Serena. Chile, (s/d), Disponível em: http://www.ctio.noao.edu/light_pollution/efectos.html. Acesso em: 09 fev. 2012.

MIRANDA, P. D. Discurso del representante de CONAMA. Light Pollution: The Global View. SCHWARZ, H.E.- Editor. Kluwer Academic Publishers. 201 p., 2003.

MURIANA, J.I. Comunicação pessoal relativa às leis existentes na Espanha 2008a.

MURIANA, J.I. La prevención de la contaminación lumínica a Catalunya: especial referencia als munícipes. Tarragona. Publicacions URV, 151 p., 2008b.

NARISADA, K., SCHREUDER, D. Light Pollution Handbook. Series: Astrophysics and Space Science Library, Springerardcover Ed., Dordrecht, Holanda, 927 p., 2004.

NETRESIDUOS. Legislação Portuguesa de Resíduos Elétricos e Eletrônicos. Decreto-Lei n. ${ }^{\mathbf{0}}$ 230/2004; n. ${ }^{0174 / 2005}$; Despacho Conjunto n. ${ }^{\circ}$ 353/2006. Disponível em:

http://www.netresiduos.com/pt/default.asp?id=1569\&mid=226 Acesso em: 11 jun 2007.

NEVES, A.I. Consultor ambiental da empresa Ambiente \& Qualidade, depoimento pessoal arquivado, 2007. 
REITER, R.J. Melatonin: the chemical expression of darkness. Molecular and Cellular Endocrinology. Elsevier, v.79, n.1-3, p. 153-158, 1991.

SALMON, M.; TOLBERT, M; PAINTER, G. D. P. Behavior of loggerhead sea turtles on an urban beach. II. Hatchling orientation. Journal of Herpetology, v. 29, n. 4, p. 568-576, 1995.

SANHUEZA, P.A.; SANTANDER, M.P. Protecting the night sky of northern Chile: An environment and cultural heritage. In: Ed. SCHWARZ, H.E. Light Pollution: The Global View. Kluwer Academic Publishers. 201 p., 2003.

SCHWARTZ, A; HENDERSON, R.W. Amphibians and reptiles of the West Indies: descriptions, distributions, and natural history. Publicado pela University of Florida Press., Gainesville, Flórida, Estados Unidos, 714 p., 1991.

SMITH, M. Time to turn off the lights. Nature, v. 457, n.1, p. 27. 2009.

TARSIA, R. D. Comunicação pessoal relativa à Lei Municipal de Caeté, MG, 2007.

WALKER, M. F. The California Site Survey 1965-1967. Publicação da The Astronomical Society of the Pacific, San Francisco, Estados Unidos, 196 p., 1970. 